\title{
Experimental Models of Abdominal Aortic Aneurysms
}

\author{
Janice C. Tsui* \\ Division of Surgery \& Interventional Science, University College London, Royal Free Campus, Pond Street, London \\ $N W 32 Q G, U K$
}

\begin{abstract}
Despite being a leading cause of death in the West, the pathophysiology of abdominal aortic aneurysms (AAA) is still incompletely understood. Pharmacotherapy to reduce the growth of small AAAs is limited and techniques for repairing aneurysms continue to evolve. Experimental models play a key role in AAA research, as they allow a detailed evaluation of the pathogenesis of disease progression. This review focuses on in vivo experimental models, which have improved our understanding of the potential mechanisms of AAA development and contributed to the advancement of new treatments.
\end{abstract}

Keywords: Abdominal aortic aneurysms (AAA), experimental models, elastase infusion.

\section{INTRODUCTION}

Abdominal aortic aneurysm (AAA) has a prevalence of $5 \%$, accounting for over 11,000 hospital admissions and 10,000 deaths per year in the UK [1]. It affects primarily older males where ruptured AAA results in approximately $2 \%$ of total mortality in men over 65 years of age [2]. Other risk factors include family history, hypertension, hypercholesterolaemia, atherosclerosis and cigarette-smoking [3, 4].

Whilst most AAAs are asymptomatic, expanding aneurysms can cause pressure symptoms and more significant symptoms include distal embolisation and rupture. Patients with free intraperitoneal rupture rarely reach hospital alive and for those who have surgery, perioperative mortality is approximately $50 \%$ [1].

The mainstay of AAA management is therefore elective repair prior to rupture. Based on results of the MRC small aneurysm trial where 30-day mortality from elective surgery was $5.8 \%$, AAAs of less than $5.5 \mathrm{~cm}$ diameter are managed conservatively whilst those above this size are considered for repair [5]. Methods of repair consist predominantly of open or endovascular repair. There is evidence that for those who are suitable for endovascular repair, this procedure is associated with reduced morbidity and mortality [6].

For patients with small AAAs $(<5.5 \mathrm{~cm})$, aggressive risk factor management is required to reduce cardiovascular morbidity and mortality. Pharmacotherapy to reduce AAA growth rate remains limited, although statins have been shown to be effective [7].

Despite the clinical importance of AAAs, the aetiology and pathogenesis involved remain unclear. Histological analyses of aneurysm wall sampled at surgery demonstrate a loss of medial elastin and smooth muscle with excessive deposition of adventitial collagen and arterial wall thickening

*Address correspondence to this author at the Division of Surgery \& Interventional Science, University College London, Royal Free Campus, Pond Street, London NW3 2QG, UK; Tel: (+44) 2077940500 ext 33938; Fax: (+44) 2074726278; E-mail: j.tsui@medsch.ucl.ac.uk
[8]. However, these observations represent late changes that occur and do not necessarily reflect processes involved in disease development.

In vitro models have been used in AAA research both to investigate pathophysiology and to assess new technologies. Thompson et al. described a model of aortic organ culture where $1 \mathrm{~cm}^{2}$ segments of porcine thoracic aorta were preincubated in pancreatic elastase for 24 hours prior to standard organ culture conditions for 14 days $[9,10]$. Aortic extracellular matrix degradation and increases in matrix metalloproteinases (MMP) expression were found. When autogenous leucocytes were added to the culture after 48 hours of elastase incubation, an inflammatory infiltrate developed and enhanced the matrix degradation that occurred. Further modifications of this model included culturing under laminar shear stress or static conditions. Whilst this was a useful model, the isolated aortic segments could only be maintained in culture for a limited period and the model may not reflect events that occur in vivo. For the investigation of novel techniques, in vitro models of varying sophistication have been described, using either synthetic materials or a combination of synthetic and biological materials [11-13]. These play an important role in "proof of concept studies" but require further validation prior to clinical use.

This underscores the importance of in vivo animal models; enabling researchers to test hypotheses relating to AAA pathogenesis and to investigate potential pharmacotherapy. They have also played a key role in the development of new interventions for the treatment of AAAs with the aim of reducing peri-operative morbidity and mortality.

This review will outline some of the animal models that have been used, focusing on the contributions they have made to our understanding and management of AAAs.

\section{ANIMAL MODELS}

Animal models can be broadly classified into three categories: genetically predisposed animal models, chemical models and physical models. 


\section{Genetically Predisposed Animal Models}

Early in vivo studies of AAA relied on animals with susceptibility to atherosclerosis and aneurysm formation. The Broad Breasted Bronze and American Mammoth Bronze breeds of turkeys were noted to develop rupture of dissecting aneurysms [14]. Manipulation or supplementation of their feeds with various compounds altered the risk of rupture. For example, feeding these animals beta-aminoproprionitrile, the active agent in sweet pea meal, increased the risk of rupture [15] and the effects were further exacerbated by diethylstilbestrol, monoamine oxidase inhibitors, and hydralazine [1618]. The latter was found not to act via its haemodynamic effects but by accumulating in the aortic media and reacting with collagen groups. In contrast, propranolol and reserpine decreased the risk of aortic rupture [17, 19]. Whilst both these agents altered haemodynamics, propanolol also increased elastin cross-linking [15, 18]. These early studies established the involvement of haemodynamic factors and connective tissue changes in the development and rupture of aortic aneurysms.

The blotchy mouse was also found to develop spontaneous anuerysms [20]. These mice have a genetic abnormality on the $\mathrm{X}$ chromosome which results in defective collagen and elastin cross-linking. They develop abnormalities in connective tissue, skin colour and neurological function, due to an underlying defect in copper metabolism [21]. They also develop saccular and fusiform aneurysms throughout the aorta, but predominantly in the thoracic aorta [20]. Propranolol delayed aneurysm formation probably by a combination of haemodynamic effects and increased elastin and collagen cross-linking [22, 23], whilst hydrocortisone induced aortic rupture in these mice [24]. Based on these findings, altered copper metabolism was proposed to contribute to aneurysm development in humans, however, no change in copper levels in aortic tissue from patients with AAA have been reported $[25,26]$.

Whilst these animals were useful models and increased our early understanding of the pathophysiology of aneurysms, genetically altered mice have been more valuable for the study of specific molecular pathways involved. For example, mice with a genetically engineered deficiency in lysyl oxidase (Lox), which is important in the crosslinkage of elastin and collagen, die in the perinatal period of ruptured thoracic aortic aneurysms [27]. Apolipoprotein E (ApoE) and low-density lipoprotein (LDL) receptor knockout mice fed on high-fat diets develop suprarenal aortic aneurysms [28]. Tsukuba hypertensive mice produced by cross-mating transgenic mice carrying either the human renin or human angiotensinogen genes [29] developed chronic hypertension and died of ruptured aortic aneurysms when fed drinking water containing $1 \%$ sodium chloride. The aneurysms were found to be predominantly located at the aortic arch or the juxtarenal segment of the abdominal aorta [30]. Mice with MMP-3 or tissue inhibitor of MMP (TIMP)-1 deficiencies develop aneurysms in both thoracic and abdominal aortas $[31,32]$. Some of these mice however are not suitable experimental models since they died early of ruptured aneurysms or developed aneurysms at atypical locations. Others have been successfully used in chemical models as described below.

\section{Chemical Models}

Models where aneurysms have been induced by the application of chemical agents have been useful in elucidating the aetiology and molecular mechanisms of aneurysm formation. They are also useful tools for testing potential pharmacotherapy in AAA management.

Extraluminal application of calcium chloride $\left(\mathrm{CaCl}_{2}\right)$ solution to arteries results in aneurysm formation. This was first described in a study using rabbit common carotid artery, where progressive focal dilatation of the artery occurred, with loss of endothelial cells, disruption of the internal elastic lamina, intimal hyperplasia and inflammatory infiltration of the media [33]. This method was then applied to rabbit aortas, where $2 \mathrm{~cm}$ of proximal infrarenal aorta was dissected out and $\mathrm{CaCl}_{2}$ applied with a cotton wool tip for 15 minutes [34]. $0.25 \mathrm{ml} / \mathrm{L} \mathrm{CaCl}_{2}$ was found to cause medial damage with vascular remodelling, reduction in insoluble collagen and increased gelatinases activity, leading to aneurysm formation. Inflammation induced by thioglycollate together with diet-induced hypercholesterolaemia led to rapid aneurysmal dilatation. A murine model was also successfully created using a similar technique. Aortas increased by $64 \%$ in diameter 2 weeks after treatment and by $110 \% 3$ weeks after treatment [35]. Aneurysms formed demonstrated disruption of the media elastic network and activation of the inflammatory response as seen in human AAAs, suggesting that this is a clinically useful model. A murine model offers the advantage of working with mice, which are low cost, easy to handle, have a short life cycle as well as a wellstudied genome suitable for genetic manipulation.

Using this model in genetically altered mice, the role of MMPs in AAA formation has been established: MMP2 -/and MMP9 -/- knockout mice did not form aneurysms [36], whilst MMP12 -/- mice showed attenuated aortic dilatation compared to wild-types following $\mathrm{CaCl}_{2}$-mediated injury [37]. As the MMPs are tightly regulated by the TIMPs, their role has also been studied. Surprisingly, in mice deficient in TIMP-2, $\mathrm{CaCl}_{2}$-mediated injury resulted in significantly smaller aneurysms than wild-types at 6 weeks [38], suggesting that there is a complex interaction between the MMPs and TIMPs. The hypothesis that chemokines are involved in neutrophil infiltration of the aortic wall in aneurysm development was tested using mice with genetic deletions of the chemokine receptors CCR2, CCR5 and CXCR3. Six weeks after $\mathrm{CaCl}_{2}$-mediated injury, aneurysm formation was attenuated in CCR2 -/- mice with reduced inflammatory infiltration whilst CCR5 and CXCR3 deletion conferred no protection against aneurysm formation [39].

Agents that may reduce aneurysm formation or growth have been studied using this model. For example, daily oral doses of the anti-inflammatory agent indomethacin following $\mathrm{CaCl}_{2}$ application resulted in less disruption of the elastic layers within the aortic wall and reduced aortic dilatation three weeks after surgery in rats [40]. The effect of pentagalloyl glucose (PGG), a polyphenolic tannin, on aneurysm formation and growth in rats, has also been explored [41]. Pre-treating rat aortas with $\mathrm{PGG}$ prior to $\mathrm{CaCl}_{2}$ application inhibited aneurysm formation whilst applying PGG to aneurysms formed 28 days after $\mathrm{CaCl}_{2}$ injury prevented further aneurysm growth. PPG was found to bind to aortic elastin, 
preventing elastin degradation despite high levels of MMPs [41].

The elastase-induced model is another important model, first described by Anidjar et al. [42]. The procedure involved dissecting out the rat abdominal aorta followed by insertion of a catheter via the femoral artery into the infrarenal aorta. The aorta was then clamped at the level of the left renal vein and ligated around the catheter $1 \mathrm{~cm}$ distally. This segment of isolated aorta was then perfused with $2 \mathrm{ml}$ of fluid at $1 \mathrm{ml} /$ hour. After 2 hours, the aorta was unclamped, the suture and catheter removed and the femoral artery ligated. Perfusion of hog pancreatic elastase resulted in destruction of the media elastin network with immediate aortic dilatation followed by aneurysm formation over 2-5 days. These findings supported the hypothesis that elastolytic activity within the media plays a role in aneurysm formation and provided a useful reproducible model for further investigations.

Using this model, documented risk factors for AAA were investigated. The role of hypertension in AAA growth was examined by Gadowski et al. by inducing aneurysms in normotensive Wistar-Kyoto rats (WKY) and genetically hypertensive Wistar-Kyoto rats (WKHT) [43]. Initially, aortic diameters were the same but by day 7 , WKHT rats had larger aneurysms than normotensive rats (mean diameter 2.52 vs. $2.31 \mathrm{~mm}$ ) and at day 14 , the difference was even greater (3.45 vs. $2.36 \mathrm{~mm})$. Growth rates were also significantly increased in WKHT rats and showed strong positive correlation to systolic blood pressure. This study confirmed the role of hypertension in AAA expansion.

In order to study the role of gender, Upchurch's group investigated the effect of gonadal hormone manipulation on aneurysm diameter in rats 14 days following elastaseinfusion [44]. They found that experimental aneurysm diameter decreased in male rats treated with exogenous oestrogen, decreased in orchidectomised males, increased in surgically castrated males given hormone replacement but decreased in castrated females on hormone replacement therapy. Altered macrophage numbers in the aortic walls of these animals were found, suggesting that gonadal hormones differentially regulated AAA growth by altering macrophage infiltration.

Epidemiological studies have shown that smokers have a 3- to 6-fold increase in AAA-related death [45]. Bergoeing et $a l$. demonstrated that this was likely to be a causal relationship by exposing mice to cigarette smoke in special cages 2 weeks before inducing aortic aneurysms using the elastaseinduction model [46]. Exposure to cigarette smoke was continued for 2 weeks until the end of the experiment. In mice not exposed to cigarette smoke, $0.16 \mathrm{U} / \mathrm{ml}$ of pancreatic elastase resulted in aneurysm formation in $100 \%$ whilst a lower dose of $0.07 \mathrm{U} / \mathrm{ml}$ only caused aneurysms in $63 \%$ of mice. In contrast, when exposed to cigarette smoke, $85 \%$ of mice developed aneurysms following $0.07 \mathrm{U} / \mathrm{ml}$ of elastase and the aneurysms were $60 \%$ larger in size. Cigarette smoke increased aortic elastic degradation in these aneurysms without affecting aortic MMP levels.

As with the $\mathrm{CaCl}_{2}$ model, the use of this model in genetically modified mice has elucidated some of the key molecular mechanisms involved in aneurysm formation. Aneurysm induction by elastase infusion was inhibited in MMP9 -/- and mice deficient in both MMP9 and MMP12. In contrast, MMP12 -/- mice were not resistant to aneurysm formation. Transplantation of bone marrow cells from wild-type mice into irradiated MMP9 -/- mice resulted in aneurysm formation. The study confirmed the importance of MMP9 in aneurysm formation in this model and also suggested that infiltrating inflammatory cells were an important source of MMP9 [47]. The role of TIMP-1 was also demonstrated using this model: TIMP1 -/- mice developed larger postinfusion aortic diameters and significantly larger aneurysms at day 14 compared to wild-types [48].

Interesting findings regarding the role of the nitric oxide (NO) pathway have also been demonstrated using the elastase infusion model. In wild-type animals, nitric oxide synthase (NOS) 1 and 3 expression in aneurysms was reduced, whilst NOS 2 expression was increased, localised to infiltrating inflammatory cells. This suggested that NOS 2 may be involved in the inflammatory component of aneurysm development. However, in male NOS2 -/- mice the incidence and size of aneurysms formed were unaffected whilst in female NOS2 -/- mice, increased incidence and size of aneurysms occurred. This latter effect was prevented by previous oophrectomy, suggesting an interaction between NOS2 and gonadal hormones [49].

The role of the proinflammatory cytokine interleukin-6 (IL-6) has also been investigated using this model. In mice with targeted deletion of IL-6, suppression of aneurysm formation occurs with reduced inflammatory response [50].

Important information on potential pharmacotherapy for AAA has been gained from the elastase infusion model. Indomethacin given daily for 7 days following elastase infusion in rats was again found to inhibit aneurysm formation [51]. The effect of statins independent of their cholesterollowering properties was studied using this model in rats, since statins do not lower cholesterol in these animals. Simvastatin given by daily gastric gavage starting the day before surgery led to reduced aneurysm size 7 days following elastase infusion with downregulation of genes involved in inflammation, extracellular matrix remodelling and oxidative stress response [52]. Atorvastatin was also found to significantly reduce aneurysm size at 4 weeks, with evidence of reduced macrophage recruitment, MMP-12 expression and increased aortic wall collagen and elastin content [53]. Propanolol was found to reduce the size of elastase-induced aneurysms in hypertensive rats but had no effect on normotensive rats, suggesting that its effect was predominantly via its blood pressure lowering properties [54]. In contrast, the calcium channel antagonist nifedipine was found to reduce aneurysm size progression without affecting blood pressure or heart rate following elastase infusion in rats [55]. The angiotensin-converting enzyme (ACE) inhibitors captopril, lisinopril and enalopril all reduced aneurysm size independent of haemodynamic effects in rats 14 days after elastase infusion with reduced medial elastin degradation and inflammation. In addition, angiotensin (Ang) II (AT1) receptor antagonist losarten did not affect aneurysm size compared to control treatment [56]. However, in another study, an alternative AT1 receptor antagonist valsartan, given following elastase infusion in rats significantly reduced aneurysm development without affecting blood pressure [57]. Given the likely involvement of MMPs in aneurysm patho- 
genesis, the effects of MMP-inhibiting tetracylines have been evaluated. Doxycycline was found to inhibit aorta dilatation in a dose- dependent manner, by reducing medial elastin degradation [58].

Innovative strategies have also been investigated using this model. For example, Nakashima et al. used decoy oligodeoxynucleotides to inhibit the transcription factors NFKB and ets in rat and rabbit elastase infusion models [59, 60], whilst Miyama et al. applied biodegradable gelatin hydrogel sponge incorporating basic fibroblast growth factor over the aortas of rats [61]. Both these strategies inhibited AAA progression.

Problems with and modifications of these techniques have been described by investigators. Carsten et al. were concerned that the sizes of the aneurysms produced by Anidjar's technique were inconsistent and may be related to variations in elastase preparations. They also found that long infusion periods were required, which were associated with higher rates of complications such as lower limb ischaemia [62]. Others found that aneurysm formation rates may be low following $\mathrm{CaCl}_{2}$ injury [41]. Recently, Tanaka et al. described a rat model which combined a 20-minute infusion of elastase with extraluminal application of $\mathrm{CaCl}_{2}$ (Fig. 1) [63]. In a series using 71 rats, they had a high success rate of AAA formation of $92.7 \%$ with no complications and aneurysms formed showed inflammatory responses, elastolytic activities and MMP expression. However, saccular aneurysms were predominantly formed and like the other chemical models, the aneurysms were not associated with atherosclerosis and intraluminal thrombosis as seen in human AAAs.

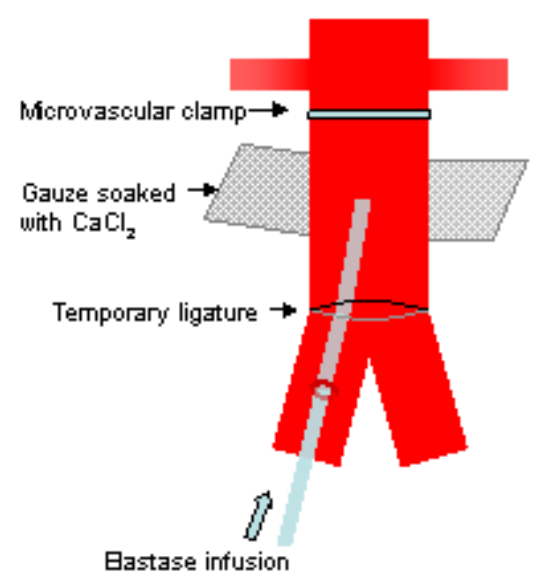

Fig. (1). Schematic drawing of experimental AAA model using a combination of intraluminal elastase infusion and extraluminal calcium chloride exposure [63].

Whilst rodents provide valuable experimental models, their small size renders them challenging to operate on. A fusiform rabbit carotid artery model created by infusing elastase into a $2 \mathrm{~cm}$ segment of the common carotid artery has been described [64]. This model offered accessibility to the aneurysm; however its size was still not suitable for the pur- pose of testing new techniques of aneurysm exclusion. The use of the elastase-perfusion technique in larger animals has been less successful: canine models have generally not been reproducible despite various protocols of elastase or elastasecollagenase infusion [65]. A study using Yucatán miniature swine did not create any aneurysms despite destruction of media elastic network following elastase infusion [66]. Recently, a porcine model has been successfully created using a combination of elastase and collagenase infusion and mechanical dilation [67]. This will be discussed below together with the physical models.

Manning et al. [68] used systemic Ang II infusion in their model. Here, Ang II was infused by subcutaneous osmotic pumps into ApoE -/- or LDL receptor -/- mice. This resulted in accelerated atherosclerosis and development of aneurysms in the suprarenal portion of the aorta, which demonstrated many of the characteristics of human disease, such as medial degeneration and remodelling, inflammation and thrombosis. Male mice were more susceptible to AAA development than females. Microarray gene analyses of aortic aneurysms confirmed that upregulation of inflammatory and extracellular matrix remodelling pathways occur, as seen in human AAAs [69]. The effects of pharmacological, surgical and genetic manipulation on AAA development in this model have been investigated [70]. Hydralazine lowered systolic blood pressure but had no effect on aneurysm formation in Ang II infused ApoE-/- mice showing that aneurysm formation in this model was independent of blood pressure [71]. 17 betaoestradiol pre-treatment reduced the incidence and severity of aneurysms in male ApoE -/- mice infused with Ang II [72], whilst dihydrotestosterone increased aneurysm incidence in castrated male and female Ang II infused ApoE -/mice, which was associated with increased AT1 receptor mRNA expression in aortas [73]. The effect of androgens on aneurysm development was supported by experiments showing reduced aneurysm incidence and severity in orchidectomised male ApoE -/- mice infused with Ang II [74]. Various agents were used to investigate further the role of the renin-angiotensin system in this model: co-infusion of the AT1 receptor antagonist losarten with Ang II in ApoE -/mice completely inhibited aneurysm formation whilst coinfusion with the AT2 receptor antagonist PD123319 increased the incidence and severity of aneurysms [75]; candesarten, another AT1 antagonist and lisinopril, an ACE inhibitor, given after Ang II infusion in ApoE -/- mice attenuated expansion of aortic aneurysms [76]; pre-treatment with the aldosterone receptor antagonist spironolactone had no effect on aneurysm formation [77]. Doxycycline was found to reduce the incidence and severity of aneurysm formation in Ang II infused LDL receptor -/- mice [78]; vitamin E attenuated aneurysm formation and size of aneurysms formed in Ang II treated ApoE-/- mice [79]; simvastatin reduced the incidence and extent of aneurysm formation with reduced remodelling and inflammatory response seen in aortic walls [80]; and rosiglitazone reduced the maximum size of the aneurysms formed and inhibited rupture when given before or after Ang II infusion in ApoE-/- mice [81].

Combining this model with genetically altered animals has also yielded interesting results. For example, the role of urokinase plasminogen activator (UPA) in aneurysm formation in this model was demonstrated using mice deficient in 
both ApoE and UPA where the incidence of aneurysm formation was reduced compared to in ApoE -/- mice [82]. Furthermore, local overexpression of the key inhibitor of UPA, plasminogen activator inhibitor-1 (PAI-1) in ApoE -/- mice using intra-adventitial injections of recombinant adenovirus expressing the human PAI-1 gene prior to Ang II infusion inhibited aneurysm formation, whilst administration of the gene 2 weeks after Ang II infusion prevented expansion of small aneurysms [83]. As increased cyclooxygenase (COX)2 expression has been found in human AAAs, COX-2 deficient mice were also studied and found to have significantly reduced incidence of aneurysm formation, associated with reduced inflammatory cell infiltration of the aorta following Ang II infusion compared to wild-types [84]. These studies demonstrate the value of combining genetically altered animals with pharmacological models of AAA formation.

\section{Physical Models}

A range of physical techniques have been used to create aneurysms in animals for investigation. However, since the pathology of these models does not reflect that of true aneurysms in humans, their use is mainly limited to the assessment of new interventions for AAA repair. With the advent of endovascular aneurysm repair (EVAR) techniques described by Parodi in 1991[85], these models have been valuable in the development of stent grafts, resulting in improved designs, delivery systems and understanding of specific complications of endovascular repair.

Crude methods of inducing aortic aneurysms included traumatising the vessel wall with intramural injections of chemicals such as acetrizoate [86] or physical damage using cryogenic probes, carbon dioxide lasers or simply surgically resecting the media and adventitia of the aorta [87-89]. These methods generally produced aneurysms of unpredictable size, growth rate and rupture risk and more often resulted in saccular aneurysms or even pseudoaneurysms.

Synthetic aneurysm models included the replacement of a segment of aorta with interposition grafts made of synthetic or autologous materials. These produced aneurysms of more consistent shape and size but do not preserve lumbar and mesenteric collateral circulation to the sac. Parodi and Palmaz's group replaced a segment of infrarenal aorta with a fusiform synthetic aneurysm made of woven Dacron in dogs to evaluate an early stent graft made of Dacron and balloonexpandable stents. This early study showed that the technique was feasible and identified problems related to device introduction, deployment and fixation [90].

Whitbread et al. created a model by interpositioning a fusiform segment of glutaraldehyde-tanned bovine internal jugular vein into the infrarenal aortas of large white pigs [91]. The resulting aneurysm had an aorta to aneurysm diameter ratio of 1:2. These aneurysms were stented 2 weeks later using plain 48-wire Wallstents (Schneider Stent, Inc., USA) via the left common carotid artery. Pigs were followed up for 6 weeks and assessed with ultrasound, angiography, pressure measurements and finally sacrificed for histology. Three out of 6 aneurysms were successfully excluded immediately whilst the remaining 3 were partially treated with reduction in aneurysm size and sac pressure. In 2 cases, the stent was inadvertently deployed over the renal arteries but no flow disturbance was found on follow-up. The authors suggested that stents could therefore be designed with a central covered portion flanked by uncovered portions for fixation. This study not only presented a new AAA model and confirmed the potential of endovascular techniques but also contributed to advancement in stent graft design.

The anterior patch model is another successful physical model, where following a longitudinal aortotomy, an elliptical patch of either autologous or synthetic material was used to close the aortotomy. For example, Criado et al. created infrarenal aortic aneurysms by suturing full thickness jejunal patches to longitudinal anterior aortotomies in dogs to test the longer term efficacy of endovascular aortic repair [92]. Six out of 7 untreated aneurysms ruptured within 6 days. In $11 \mathrm{dogs}$, the aneurysm was stented using an $8 \mathrm{~mm}$ Dacron graft introduced via the femoral artery. Dogs were followed up with pressure measurements, angiography and histology when sacrificed at 3 and 6 months. Three aneurysms were not excluded successfully and ruptured within 4 days. Two dogs from the earlier part of the study became paraplegic due to stent kinking and thrombosis. The remaining aneurysms were successfully excluded with incorporation of graft material into the surrounding tissue, which was evident at 3 months with no evidence of graft migration.

Eton et al. also used an autologous anterior patch model in mongrel dogs to assess a specific stent graft design and delivery system [93]. Here, an iliac vein patch was used to form fuisform infrarenal aneurysms. This model was more realistic as the lumbar and mesenteric branches were preserved, allowing the effect of patent side-branches on subsequent aneurysm exclusion and size to be studied. Twenty three dogs with aneurysms were treated with Dacron stent grafts and followed up for 6 to 12 months. At 12 months, there were no leaks or flow within the sac and no sac enlargement, confirming the feasibility of this technique despite the presence of patent side branches.

Whilst the benefits of EVAR include reduced 30-day mortality, better early health-related quality of life and improved long-term aneurysm-related survival, EVAR is associated with significantly increased secondary intervention rate $[6,94]$. Advances in stent graft technology have reduced specific complications such as graft migration and thrombosis, but endoleaks remain causes of re-intervention. Endoleaks result in continued pressurisation of the aneurysm sac due to persistent flow outside the stent graft. They occur in up to $26 \%$ of patients following EVAR [95] and may lead to aortic rupture [96]. Endoleaks due to incompetent seal at attachment sites (type I) and between modular components (type III) result in incomplete exclusion of the aneurysm and require early intervention. These have been reduced with improved graft design and planning. Type IV endoleaks occur through porous graft material and are now rarely seen. Type II endoleaks result from flow into and out of the aneurysm sac from patent side-branches such as the inferior mesenteric or lumbar arteries (Fig. 2). Whilst it was recognised that Type I and III endoleaks required urgent treatment to prevent aortic rupture, the clinical significance of type II endoleaks were less clear.

In order to study the significance of type II endoleaks, specific models were developed by some groups. Mousa et al. modified a prosthetic model in dogs by dilating $8 \mathrm{~mm}$ polytetrafluroethylene (PTFE) grafts with a $30 \mathrm{~mm}$ - 
A

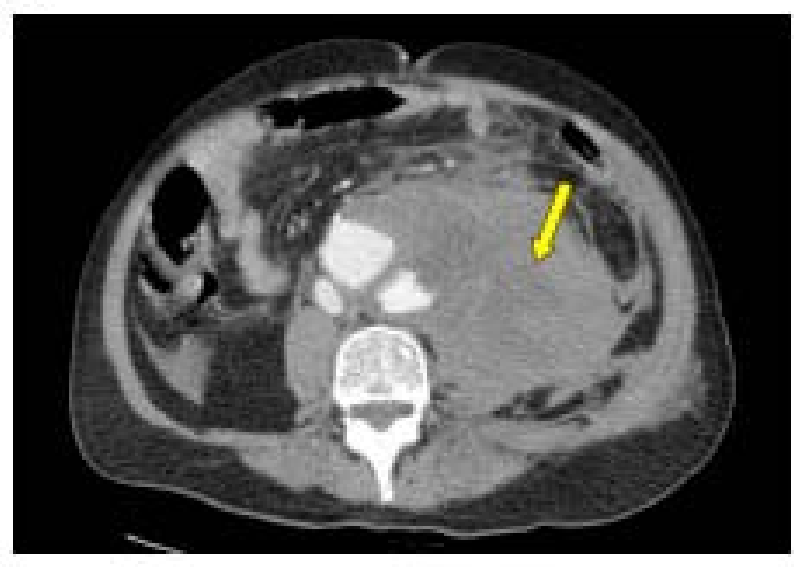

B

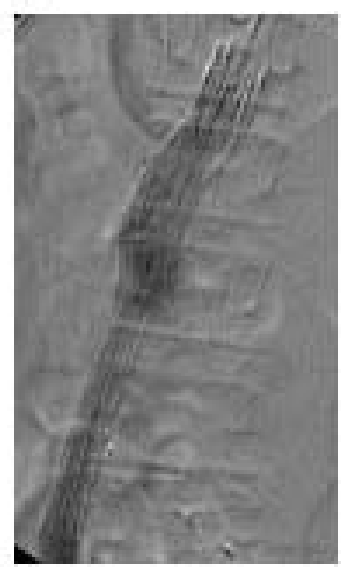

C

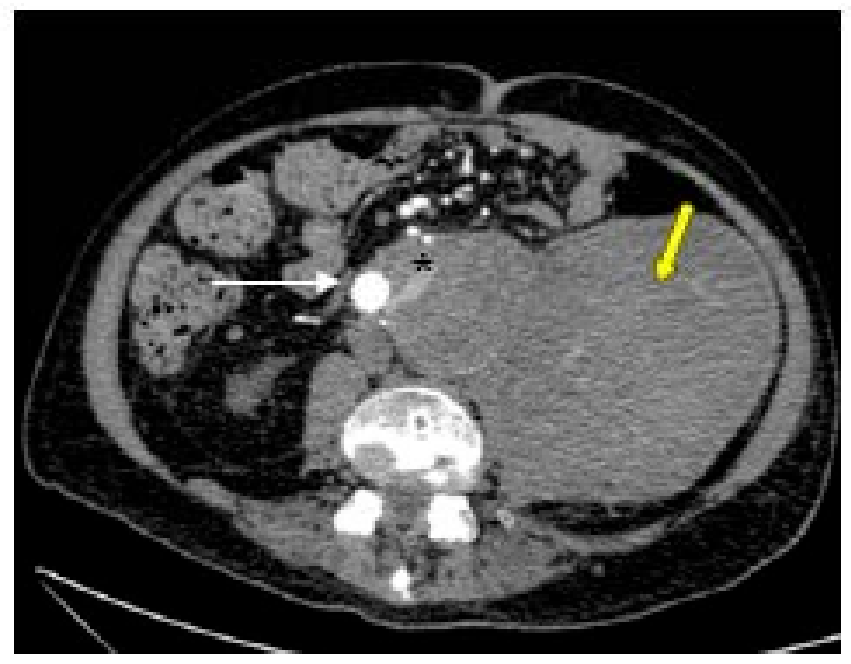

Fig. (2). A: CT of a ruptured AAA in a 72 year old man with a large haematoma (yellow arrow) B: Intra-operative angiogram during urgent EVAR showing stent graft in position, excluding the AAA C: Post-EVAR CT showing stent graft in situ and type II endoleak. Contrast is seen within the sac outside of the stent graft ( white arrow $=$ stent graft, ${ }^{*}=$ endoleak), arising from one of the lumbar arteries. The haematoma caused at the time of rupture is still present (yellow arrow).

angioplasty balloon and incorporating intraluminal pressure transducers to monitor sac pressures [97]. At implantation, a segment of native aorta with 2 or more patent side branch vessels (lumbar +/- caudal mesenteric arteries) were reimplanted onto the posterior aspect of the prosthetic aneurysm. Before endovascular exclusion with a PTFE-covered stent graft, pressures within the aneurysm sac closely matched systemic pressures. In models where no side branches were reimplanted, endovascular stenting resulted in significant reduction in sac pressures and no endoleak. In the presence of patent side branches, endovascular stenting resulted in a smaller fall in sac pressures and type II endoleaks were demonstrated on angiography. The side branches remained patent on duplex and magnetic resonance angiography (MRA) throughout the study period of 90 days. This study demonstrated that type II endoleaks resulted in significant sac pressure and if persistent may be of clinical significance. However, the main limitation of this study was the use of relatively nonexpansile prosthetic material to create aneurysms which were unlikely to simulate post-stenting aneurysms.
Diaz et al. used a porcine anterior patch model where a $4 \mathrm{~cm}$ wide ellipse of Dacron was sutured to an infrarenal aortotomy [98]. Two lumbar arteries were preserved in all the animals and pressure transducers were implanted in the aneurysm wall. One week later, EVAR was performed with a custom-made stent graft. All the aneurysms were excluded with type II endoleaks via the lumbar arteries. Following EVAR, mean arterial pressure within the aneurysm sac decreased but pulse pressure persisted. A further week later, laparoscopic lumbar artery ligation was performed via a retroperitoneal approach which resulted in further reduction in mean arterial pressure and obliteration of pulse pressure within the aneurysm sac. Successful treatment of the type II endoleak was confirmed in all animals on CT scans and on histological analyses. This study showed that type II endoleaks were associated with persistent pulse pressure within the sac and that both sac pulse pressure measurements and CT scans were useful in identifying type II endoleaks. It also demonstrated that laparoscopic ligation of side branches was an effective way of treating type II endoleaks. The in 
vivo porcine model created may provide a useful tool for the development and training of this technique. Unfortunately, the study period was short which did not allow for the natural history of type II endoleaks to be studied: it is now recognised that the majority of type II endoleaks resolve spontaneously within 6 to 12 months and do not require intervention [95].

More recently, Maynar et al. described a version of the anterior patch model in swine where a $2-3 \mathrm{~mm}$ wide segment of the aortic wall was resected following anterior aortotomy, and a double-layered oval peritoneal patch sutured to close the defect [99]. Aneurysm development in these animals was followed up with angiography as well as laparoscopic assessment and laparoscopic ultrasound and finally histological analyses. The infrarenal aneurysms created increased in size up to 30 days post-operatively and 15 out of 27 aneurysms ruptured within 2 weeks. Laparoscopic assessment showed incorporation of patches into the aortic wall with almost complete endothelialization of the luminal wall. The authors argued that this model was more realistic than others due to its potential for aneurysmal growth and rupture. However, like other anterior patch models, the incorporation of biological material into the aortic wall limited its use in the study of tissue response to stent graft placement. Furthermore, whilst the side branches were preserved in this model, these arose from normal rather than aneurysmal aortic wall as seen in patients with AAAs.

In order to overcome some of these problems, a previously mentioned porcine model using a combination of mechanical aortic dilation and enzymatic connective tissue degradation has been described by Hynecek [67]. A segment of infrarenal aortic was dissected out and gradually dilated with a non-compliant angioplasty balloon of $12-14 \mathrm{~mm}$ diameter introduced proximal to the caudal mesenteric artery. This segment of aorta was then isolated with vascular clamps and infused with $50 \mathrm{ml}$ of a solution of collagenase and elastase under pressure for 20 minutes. The aortic lumen was irrigated to remove remaining solution prior to closing the arteriotomy. Aneurysm formation in 10 pigs was assessed with serial MRAs and histological evaluation when the animals were euthanised. Following mechanical dilation, there was a mean $62 \%$ increase aortic diameter and at 1,3 and 6 weeks after surgery, aortic diameters had increased by 73,83 and $94 \%$ respectively. Histologically, there was early loss of endothelial cells with gradual re-endothelialisation; mural neutrophil infiltration; loss of smooth muscle cell with limited repopulation; elastin disruption and persistent degradation and collagen disruption and degradation followed by deposition. These changes were similar to the degenerative changes that occur in human AAAs. Further work by this group has shown that gene expression in this model as demonstrated by gene arrays, replicated the patterns seen in human aneurysms, predominantly with upregulation of extracellular matrix remodelling and inflammatory genes and downregulation of elastin [100]. This model therefore represents a large animal native aneurysm model which may be useful in the further development of stent graft technology, where the behaviour of novel components within abnormal aorta can be studied. In addition, the effect of endovascular intervention on AAA pathophysiology can also be investigated. The main drawback of this model is the absence of side branches since

the lumbar arteries are ligated to ensure that the collagenase/ elastase solution does not enter the systemic circulation.

Another porcine model combined intraluminal infusion of porcine pancreatic elastase with the application of a plastic cuff below the level of the renal arteries in order to simulate turbulent flow [101]. The application of this model requires further evaluation.

\section{CONCLUSION}

In vivo models have been important in increasing our understanding of the pathophysiology of AAAs and continue to be valuable tools for the development of pharmacotherapy and new technologies for aneurysm repair. The models have limitations and appropriate choice of model according to the purpose of the study is important. Overall limitations include interspecies variation in anatomy, physiology and pharmacodynamics. For example, the anatomy of some of the larger animal models may not be suitable for assessment of stent graft delivery systems for humans and variations in fibrinolytic systems may result in increased complications in some species [102]. Experimental models do not include the complex risk factors that patients are exposed to before and after aneurysm repair. The results from in vivo models may be hard to interpret particularly as results from different models may be conflicting and findings may not be applicable to patients. Indeed, not all the potential pharmacological agents shown to be promising in animal models are effective clinically, where the main aims of pharmacotherapy are to reduce or prevent growth of small AAAs. In a recent systematic review and meta-analysis of pharmacotherapy for decreasing AAA expansion rate, statins were found to be effective whilst beta-blockers were not; ACE inhibitors, calcium channel blockers, non-steroidal anti-inflammatory agents and doxycycline may be useful but require further evaluation [7].

$\begin{array}{ll}\text { ABBREVIATIONS } \\ \text { AAA } & =\text { Abdominal aortic aneurysm } \\ \text { ACE } & =\text { Angiotensin converting enzyme } \\ \text { Ang II } & \text { Angiotensin II } \\ \text { Apo E } & =\text { Apolipoprotein E } \\ \text { AT1 } & =\text { Type } 1 \text { angiotensin II receptor } \\ \text { AT2 } & =\text { Type } 2 \text { angiotensin II receptor } \\ \text { Ca Cl } & =\text { Calcium chloride } \\ \mathrm{COX} & =\text { Cyclooxgenase } \\ \text { EVAR } & =\text { Endovascular aortic aneurysm repair } \\ \text { LDL } & =\text { Low density lipoprotein } \\ \text { Lox } & =\text { Lysyl oxidase } \\ \text { MMP } & =\text { Matrix metalloproteinase } \\ \text { MRA } & =\text { Magnetic reasonance angiography } \\ \text { NO } & =\text { Nitric oxide } \\ \text { NOS } & =\text { Nitric oxide synthase } \\ \text { PAI-1 } & =\text { Plasminogen activator inhibitor-1 } \\ \text { PPG } & =\text { Pentagalloyl glucose } \\ & \end{array}$

\section{ABBREVIATIONS}




$$
\begin{aligned}
& \text { PTFE }=\text { Polytetrafluroethylene } \\
& \text { TIMP }=\text { Tissue inhibitor of MMP } \\
& \text { UPA }=\text { Urokinase plasminogen activator } \\
& \text { WKHT }=\text { Wistar Kyoto hypertensive } \\
& \text { WKY }=\text { Wistar Kyoto rat }
\end{aligned}
$$

\section{REFERENCES}

[1] Thompson MM, Bell PR. ABC of arterial and venous disease. Arterial aneurysms. Br Med J 2000; 320: 1193-6.

[2] Crane J, Cheshire N. Recent developments in vascular surgery. BMJ 2003; 327: 911-5.

[3] Lindblad B, Borner G, Gottsater A. Factors associated with development of large abdominal aortic aneurysm in middle-aged men. Eur J Vasc Endovasc Surg 2005; 30: 346-52.

[4] Forsdahl SH, Singh K, Solberg S, Jacobsen BK. Risk factors for abdominal aortic aneurysms: a 7-year prospective study: the Tromso Study, 1994-2001. Circulation 2009; 119: 2202-8.

[5] Mortality results for randomised controlled trial of early elective surgery or ultrasonographic surveillance for small abdominal aortic aneurysms. The UK Small Aneurysm Trial Participants. Lancet 1998; 352: 1649-55.

[6] EVAR trial participants. Endovascular aneurysm repair versus open repair in patients with abdominal aortic aneurysm (EVAR trial 1): randomised controlled trial. Lancet 2005; 365: 2179-86.

[7] Guessous I, Periard D, Lorenzetti D, Cornuz J, Ghali WA. The efficacy of pharmacotherapy for decreasing the expansion rate of abdominal aortic aneurysms: a systematic review and metaanalysis. PLoS One 2008; 3: e1895.

[8] Guo DC, Papke CL, He R, Milewicz DM. Pathogenesis of thoracic and abdominal aortic aneurysms. Ann N Y Acad Sci 2006; 1085: 339-52.

[9] Thompson MM, Wills A, McDermott E, Crowther M, Brindle N, Bell PR. An in vitro model of aneurysmal disease: effect of leukocyte infiltration and shear stress on MMP production within the arterial wall. Ann N Y Acad Sci 1996; 800: 270-3.

[10] Wills A, Thompson MM, Crowther M, et al. Elastase-induced matrix degradation in arterial organ cultures: an in vitro model of aneurysmal disease. J Vasc Surg 1996; 24: 667-79.

[11] Schurink GW, Aarts NJ, Wilde J, et al. Endoleakage after stentgraft treatment of abdominal aneurysm: implications on pressure and imaging--an in vitro study. J Vasc Surg 1998; 28: 234-41.

[12] Dias NV, Ivancev K, Malina M, et al. Direct intra-aneurysm sac pressure measurement using tip-pressure sensors: in vivo and in vitro evaluation. J Vasc Surg 2004; 40: 711-6.

[13] Springer F, Schlierf R, Pfeffer JG, Mahnken AH, Schnakenberg U, Schmitz-Rode T. Detecting endoleaks after endovascular AAA repair with a minimally invasive, implantable, telemetric pressure sensor: an in vitro study. Eur Radiol 2007; 17: 2589-97.

[14] Gresham GA, Howard AN. Aortic rupture in the turkey. J Atheroscler Res 1961; 1: 75-80.

[15] Simpson CF, Kling JM, Palma RF. Beta-aminopropriomitrileinduced dissecting aneurysms of turkeys: treatment with propanalol. Toxicol Appl Pharmacol 1970; 16: 143-53.

[16] Simpson CF. Relation of hemodynamics to the incidence of diethylstilbestrol-induced aortic ruptures in hypertensive and hypotensive lines of turkeys. Atherosclerosis 1978; 30: 249-54.

[17] Simpson CF, Kling JM, Robbins RC, Harms RH. Betaaminoproprionitrile-induced aortic ruptures in turkey and inhibition by reserpine and enhancement by monoamine oxidase inhibitors. Toxicol Appl Pharmacol 1968; 12: 48-59.

[18] Simpson CF, Boucek RJ. The B-aminopropionitrile-fed turkey: a model for detecting potential drug action on arterial tissue. Cardiovasc Res 1983; 17: 26-32.

[19] Simpson CF, Kling JM, Palmer RF. The use of propranolol for the protection of turkeys from the development of betaaminopropionitrile-induced aortic ruptures. Angiology 1968; 19: 414-8.

[20] Andrews EJ, White WJ, Bullock LP. Spontaneous aortic aneurysms in blotchy mice. Am J Pathol 1975; 78: 199-210.

[21] Hunt DM. Primary defect in copper transport underlies mottled mutants in the mouse. Nature 1974; 249(460): 852-4.
[22] Brophy C, Tilson JE, Tilson MD. Propranolol delays the formation of aneurysms in the male blotchy mouse. J Surg Res 1988; 44: 6879.

[23] Brophy CM, Tilson JE, Tilson MD. Propranolol stimulates the crosslinking of matrix components in skin from the aneurysmprone blotchy mouse. J Surg Res 1989; 46: 330-2.

[24] Reilly JM, Savage EB, Brophy CM, Tilson MD. Hydrocortisone rapidly induces aortic rupture in a genetically susceptible mouse. Arch Surg 1990; 125: 707-9.

[25] Senapati A, Carlsson LK, Fletcher CD, Browse NL, Thompson RP. Is tissue copper deficiency associated with aortic aneurysms? Br J Surg 1985; 72: 352-3.

[26] Jaakkola P, Hippelainen M, Kantola M. Copper and zinc concentrations of abdominal aorta and liver in patients with infrarenal abdominal aortic aneurysm or aortoiliacal occlusive disease. Ann Chir Gynaecol 1994; 83: 304-8.

[27] Maki JM, Rasanen J, Tikkanen H, et al. Inactivation of the lysyl oxidase gene Lox leads to aortic aneurysms, cardiovascular dysfunction, and perinatal death in mice. Circulation 2002; 106: 25039.

[28] Tangirala RK, Rubin EM, Palinski W. Quantitation of atherosclerosis in murine models: correlation between lesions in the aortic origin and in the entire aorta, and differences in the extent of lesions between sexes in LDL receptor-deficient and apolipoprotein E-deficient mice. J Lipid Res 1995; 36: 2320-8.

[29] Fukamizu A, Sugimura K, Takimoto E, et al. Chimeric reninangiotensin system demonstrates sustained increase in blood pressure of transgenic mice carrying both human renin and human angiotensinogen genes. J Biol Chem 1993; 268: 11617-21.

[30] Nishijo N, Sugiyama F, Kimoto K, et al. Salt-sensitive aortic aneurysm and rupture in hypertensive transgenic mice that overproduce angiotensin II. Lab Invest 1998; 78: 1059-66.

[31] Silence J, Lupu F, Collen D, Lijnen HR. Persistence of atherosclerotic plaque but reduced aneurysm formation in mice with stromelysin-1 (MMP-3) gene inactivation. Arterioscler Thromb Vasc Biol 2001; 21: 1440-5.

[32] Silence J, Collen D, Lijnen HR. Reduced atherosclerotic plaque but enhanced aneurysm formation in mice with inactivation of the tissue inhibitor of metalloproteinase-1 (TIMP-1) gene. Circ Res 2002; 90: 897-903.

[33] Gertz SD, Kurgan A, Eisenberg D. Aneurysm of the rabbit common carotid artery induced by periarterial application of calcium chloride in vivo. J Clin Invest 1988; 81: 649-56.

[34] Freestone T, Turner RJ, Higman DJ, Lever MJ, Powell JT. Influence of hypercholesterolemia and adventitial inflammation on the development of aortic aneurysm in rabbits. Arterioscler Thromb Vasc Biol 1997; 17: 10-7.

[35] Chiou AC, Chiu B, Pearce WH. Murine aortic aneurysm produced by periarterial application of calcium chloride. J Surg Res 2001; 99: 371-6.

[36] Longo GM, Xiong W, Greiner TC, Zhao Y, Fiotti N, Baxter BT. Matrix metalloproteinases 2 and 9 work in concert to produce aortic aneurysms. J Clin Invest 2002; 110: 625-32.

[37] Longo GM, Buda SJ, Fiotta N, et al. MMP-12 has a role in abdominal aortic aneurysms in mice. Surgery 2005; 137: 457-62.

[38] Xiong W, Knispel R, Mactaggart J, Baxter BT. Effects of tissue inhibitor of metalloproteinase 2 deficiency on aneurysm formation. J Vasc Surg 2006; 44: 1061-66.

[39] MacTaggart JN, Xiong W, Knispel R, Baxter BT. Deletion of CCR2 but not CCR5 or CXCR3 inhibits aortic aneurysm formation. Surgery 2007; 142: 284-8.

[40] Karapolat S, Unlu Y, Erkut B, Kocak H, Erdogan F. Influence of indomethacin in the rat aneurysm model. Ann Vasc Surg 2006; 20 : 369-75.

[41] Isenburg JC, Simionescu DT, Starcher BC, Vyavahare NR. Elastin stabilization for treatment of abdominal aortic aneurysms. Circulation 2007; 115: 1729-37.

[42] Anidjar S, Osborne-Pellegrin M, Coutard M, Michel JB. Arterial hypertension and aneurysmal dilatation. Kidney Int Suppl 1992; 37: S61-6.

[43] Gadowski GR, Ricci MA, Hendley ED, Pilcher DB. Hypertension accelerates the growth of experimental aortic aneurysms. J Surg Res 1993; 54: 431-6.

[44] Cho BS, Woodrum DT, Roelofs KJ, Stanley JC, Henke PK, Upchurch GR, Jr. Differential regulation of aortic growth in male and 
female rodents is associated with AAA development. J Surg Res 2009; 155: 330-8.

[45] Lederle FA, Nelson DB, Joseph AM. Smokers' relative risk for aortic aneurysm compared with other smoking-related diseases: a systematic review. J Vasc Surg 2003; 38: 329-34.

[46] Bergoeing MP, Arif B, Hackmann AE, Ennis TL, Thompson RW, Curci JA. Cigarette smoking increases aortic dilatation without affecting matrix metalloproteinase-9 and -12 expression in a modified mouse model of aneurysm formation. J Vasc Surg 2007; 45: $1217-27$.

[47] Pyo R, Lee JK, Shipley JM, et al. Targeted gene disruption of matrix metalloproteinase-9 (gelatinase B) suppresses development of experimental abdominal aortic aneurysms. J Clin Invest 2000; 105: 1641-9.

[48] Eskandari MK, Vijungco JD, Flores A, Borensztajn J, Shively V, Pearce WH. Enhanced abdominal aortic aneurysm in TIMP-1deficient mice. J Surg Res 2005; 123: 289-93.

[49] Lee JK, Borhani M, Ennis TL, Upchurch GR, Jr., Thompson RW. Experimental abdominal aortic aneurysms in mice lacking expression of inducible nitric oxide synthase. Arterioscler Thromb Vasc Biol 2001; 21: 1393-401.

[50] Thompson RW, Curci JA, Ennis TL, Mao D, Pagano MB, Pham CT. Pathophysiology of abdominal aortic aneurysms: insights from the elastase-induced model in mice with different genetic backgrounds. Ann N Y Acad Sci 2006; 1085: 59-73.

[51] Holmes DR, Petrinec D, Wester W, Thompson RW, Reilly JM. Indomethacin prevents elastase-induced abdominal aortic aneurysms in the rat. J Surg Res 1996; 63: 305-9.

[52] Kalyanasundaram A, Elmore JR, Manazer JR, et al. Simvastatin suppresses experimental aortic aneurysm expansion. J Vasc Surg 2006; 43: 117-24.

[53] Shiraya S, Miyake T, Aoki M, et al. Inhibition of development of experimental aortic abdominal aneurysm in rat model by atorvastatin through inhibition of macrophage migration. Atherosclerosis 2009; 202: 34-40.

[54] Ricci MA, Slaiby JM, Gadowski GR, Hendley ED, Nichols P, Pilcher DB. Effects of hypertension and propranolol upon aneurysm expansion in the Anidjar/Dobrin aneurysm model. Ann N Y Acad Sci 1996; 800: 89-96.

[55] Tomita N, Yamasaki K, Izawa K, et al. Inhibition of experimental abdominal aortic aneurysm progression by nifedipine. Int $\mathrm{J} \mathrm{Mol}$ Med 2008; 21: 239-44.

[56] Liao S, Miralles M, Kelley BJ, Curci JA, Borhani M, Thompson RW. Suppression of experimental abdominal aortic aneurysms in the rat by treatment with angiotensin-converting enzyme inhibitors. J Vasc Surg 2001; 33: 1057-64.

[57] Fujiwara Y, Shiraya S, Miyake T, et al. Inhibition of experimental abdominal aortic aneurysm in a rat model by the angiotensin receptor blocker valsartan. Int J Mol Med 2008; 22: 703-8.

[58] Curci JA, Petrinec D, Liao S, Golub LM, Thompson RW. Pharmacologic suppression of experimental abdominal aortic aneurysms: acomparison of doxycycline and four chemically modified tetracyclines. J Vasc Surg 1998; 28: 1082-93.

[59] Nakashima H, Aoki M, Miyake T, et al. Inhibition of experimental abdominal aortic aneurysm in the rat by use of decoy oligodeoxynucleotides suppressing activity of nuclear factor kappaB and ets transcription factors. Circulation 2004; 109: 132-8.

[60] Miyake T, Aoki M, Nakashima H, et al. Prevention of abdominal aortic aneurysms by simultaneous inhibition of NFkappaB and ets using chimeric decoy oligonucleotides in a rabbit model. Gene Ther 2006; 13: 695-704.

[61] Miyama N, Sato A, Matsubara M, Watanabe T, Ikada Y, Satomi S. Inhibitory effects of a biodegradable gelatin hydrogel sponge sheet on the progression of experimental abdominal aortic aneurysms. Ann Vasc Surg 2009; 23: 224-30.

[62] Carsten CG, III, Calton WC, Johanning JM, et al. Elastase is not sufficient to induce experimental abdominal aortic aneurysms. J Vasc Surg 2001; 33: 1255-62.

[63] Tanaka A, Hasegawa T, Chen Z, Okita Y, Okada K. A novel rat model of abdominal aortic aneurysm using a combination of intraluminal elastase infusion and extraluminal calcium chloride exposure. J Vasc Surg 2009; 50: 1423-32.

[64] Reinald N, Fournier B, Naveau A, et al. Fusiform aneurysm model in rabbit carotid artery. J Vasc Res 2010; 47: 61-8.

[65] Strindberg G, Nichols P, Ricci MA, et al. Experimental modifications to a canine infrarenal aortic aneurysm model for the valida- tion of endovascular stent-grafts: an exploratory study. J Invest Surg 1998; 11: 185-97.

[66] Marinov GR, Marois Y, Paris E, et al. Can the infusion of elastase in the abdominal aorta of the Yucatan miniature swine consistently produce experimental aneurysms? J Invest Surg 1997; 10: 129-50.

[67] Hynecek RL, DeRubertis BG, Trocciola SM, et al. The creation of an infrarenal aneurysm within the native abdominal aorta of swine. Surgery 2007; 142: 143-9.

[68] Manning MW, Cassi LA, Huang J, Szilvassy SJ, Daugherty A. Abdominal aortic aneurysms: fresh insights from a novel animal model of the disease. Vasc Med 2002; 7: 45-54.

[69] Rush C, Nyara M, Moxon JV, Trollope A, Cullen B, Golledge J. Whole genome expression analysis within the angiotensin IIapolipoprotein $\mathrm{E}$ deficient mouse model of abdominal aortic aneurysm. BMC Genomics 2009; 10: 298.

[70] Daugherty A, Rateri DL, Cassis LA. Role of the renin-angiotensin system in the development of abdominal aortic aneurysms in animals and humans. Ann N Y Acad Sci 2006; 1085: 82-91.

[71] Cassis LA, Gupte M, Thayer S, et al. ANG II infusion promotes abdominal aortic aneurysms independent of increased blood pressure in hypercholesterolemic mice. Am J Physiol Heart Circ Physiol 2009; 296: H1660-65.

[72] Martin-McNulty B, Tham DM, da Cunha V, et al. 17 Beta-estradiol attenuates development of angiotensin II-induced aortic abdominal aneurysm in apolipoprotein E-deficient mice. Arterioscler Thromb Vasc Biol 2003; 23: 1627-32.

[73] Henriques T, Zhang X, Yiannikouris FB, Daugherty A, Cassis LA. Androgen increases AT1a receptor expression in abdominal aortas to promote angiotensin II-induced AAAs in apolipoprotein Edeficient mice. Arterioscler Thromb Vasc Biol 2008; 28: 1251-6.

[74] Henriques TA, Huang J, D'Souza SS, Daugherty A, Cassis LA. Orchidectomy, but not ovariectomy, regulates angiotensin IIinduced vascular diseases in apolipoprotein E-deficient mice. Endocrinology 2004; 145: 3866-72.

[75] Daugherty A, Manning MW, Cassis LA. Antagonism of AT2 receptors augments angiotensin II-induced abdominal aortic aneurysms and atherosclerosis. Br J Pharmacol 2001; 134: 865-70.

[76] Inoue N, Muramatsu M, Jin D, et al. Involvement of vascular angiotensin II-forming enzymes in the progression of aortic abdominal aneurysms in angiotensin II- infused ApoE-deficient mice. J Atheroscler Thromb 2009; 16: 164-71.

[77] Cassis LA, Helton MJ, Howatt DA, King VL, Daugherty A. Aldosterone does not mediate angiotensin II-induced atherosclerosis and abdominal aortic aneurysms. Br J Pharmacol 2005; 144: 443-8.

[78] Manning MW, Cassis LA, Daugherty A. Differential effects of doxycycline, a broad-spectrum matrix metalloproteinase inhibitor, on angiotensin II-induced atherosclerosis and abdominal aortic aneurysms. Arterioscler Thromb Vasc Biol 2003; 23: 483-8.

[79] Gavrila D, Li WG, McCormick ML, et al. Vitamin E inhibits abdominal aortic aneurysm formation in angiotensin II-infused apolipoprotein E-deficient mice. Arterioscler Thromb Vasc Biol 2005; 25: 1671-7.

[80] Zhang Y, Naggar JC, Welzig CM, et al. Simvastatin inhibits angiotensin II-induced abdominal aortic aneurysm formation in apolipoprotein E-knockout mice: possible role of ERK. Arterioscler Thromb Vasc Biol 2009; 29: 1764-71.

[81] Jones A, Deb R, Torsney E, et al. Rosiglitazone reduces the development and rupture of experimental aortic aneurysms. Circulation 2009; 119: 3125-32.

[82] Deng GG, Martin-McNulty B, Sukovich DA, et al. Urokinase-type plasminogen activator plays a critical role in angiotensin II-induced abdominal aortic aneurysm. Circ Res 2003; 92: 510-17.

[83] Qian HS, Gu JM, Liu P, et al. Overexpression of PAI-1 prevents the development of abdominal aortic aneurysm in mice. Gene Ther 2008; 15: 224-32.

[84] Gitlin JM, Trivedi DB, Langenbach R, Loftin CD. Genetic deficiency of cyclooxygenase-2 attenuates abdominal aortic aneurysm formation in mice. Cardiovasc Res 2007; 73: 227-36.

[85] Parodi JC, Palmaz JC, Barone HD. Transfemoral intraluminal graft implantation for abdominal aortic aneurysms. Ann Vasc Surg 1991; 5: 491-9.

[86] Economou SG, Taylor CB, Beattie EJ, Jr., Davis CB, Jr. Persistent experimental aortic aneurysms in dogs. Surgery 1960; 47: 21-8.

[87] Ammirati M, Ostertag H, Rabin E, Ciric I. Pathology of laserinduced experimental aneurysms. Lasers Surg Med 1988; 8: 30817. 
[88] Quigley MR, Heiferman K, Kwaan HC, Vidovich D, Nora P, Cerullo LJ. Laser-sealed arteriotomy: a reliable aneurysm model. J Neurosurg 1987; 67: 284-7.

[89] White JC, Sayre GP, Whisnant JP. Experimental destruction of the media for the production of intracranial arterial aneurysms. $\mathbf{J}$ Neurosurg 1961; 18: 741-5.

[90] Laborde JC, Parodi JC, Clem MF, et al. Intraluminal bypass of abdominal aortic aneurysm: feasibility study. Radiology 1992; 184: 185-90.

[91] Whitbread T, Birch P, Rogers S, et al. A new animal model for abdominal aortic aneurysms: initial results using a multiple-wire stent. Eur J Vasc Endovasc Surg 1996; 11: 90-7.

[92] Criado E, Marston WA, Woosley JT, et al. An aortic aneurysm model for the evaluation of endovascular exclusion prostheses. $\mathrm{J}$ Vasc Surg 1995; 22: 306-14.

[93] Eton D, Warner D, Owens C, et al. Results of endoluminal grafting in an experimental aortic aneurysm model. J Vasc Surg 1996; 23: 819-29.

[94] Becquemin JP, Kelley L, Zubilewicz T, Desgranges P, Lapeyre M, Kobeiter H. Outcomes of secondary interventions after abdominal aortic aneurysm endovascular repair. J Vasc Surg 2004; 39: 298305.

[95] Brewster DC, Jones JE, Chung TK, et al. Long-term outcomes after endovascular abdominal aortic aneurysm repair: the first decade. Ann Surg 2006; 244: 426-38.
[96] Schlosser FJ, Gusberg RJ, Dardik A, et al. Aneurysm rupture after EVAR: can the ultimate failure be predicted? Eur J Vasc Endovasc Surg 2009; 37: 15-22.

[97] Mousa A, Dayal R, Bernheim J, et al. A canine model to study the significance and hemodynamics of type II endoleaks. J Surg Res 2005; 123: 275-83.

[98] Diaz S, Uzieblo MR, Desai KM, et al. Type II endoleak in porcine model of abdominal aortic aneurysm. J Vasc Surg 2004; 40: 33944.

[99] Maynar M, Qian Z, Hernandez J, et al. An animal model of abdominal aortic aneurysm created with peritoneal patch: technique and initial results. Cardiovasc Intervent Radiol 2003; 26: 16876.

[100] Sadek M, Hynecek RL, Goldenberg S, Kent KC, Marin ML, Faries PL. Gene expression analysis of a porcine native abdominal aortic aneurysm model. Surgery 2008; 144: 252-8.

[101] Molacek J, Treska V, Kobr J, Certik B, Skalicky T, Kuntscher V, Krizkova V. Optimization of the model of abdominal aortic aneurysm - experiment in an animal model. J Vasc Res 2009; 45: $1-5$.

[102] Kostering H, Mast WP, Kaethner T, Nebendahl K, Holtz WH. Blood coagulation studies in domestic pigs (Hanover breed) and minipigs (Goettingen breed). Lab Anim 1983; 17: 346-9.

This is an open access article licensed under the terms of the Creative Commons Attribution Non-Commercial License (http://creativecommons.org/licenses/ by-nc/3.0/) which permits unrestricted, non-commercial use, distribution and reproduction in any medium, provided the work is properly cited. 